\title{
Infusion site adverse events in breast cancer patients receiving highly emetic chemotherapy with prophylactic anti-emetic treatment with aprepitant and fosaprepitant: A retrospective comparison
}

\author{
TAKASHI TSUDA ${ }^{1}$, CHISATO KYOMORI ${ }^{2}$, TAKURO MIZUKAMI ${ }^{1}$, TOMOKO TANIYAMA ${ }^{1}$, \\ NAOKI IZAWA ${ }^{1}$, YOSHIKI HORIE ${ }^{1}$, MAMI HIRAKAWA ${ }^{1}$, TAKASHI OGURA ${ }^{1}$, \\ TAKAKO EGUCHI NAKAJIMA ${ }^{1}$, KOICHIRO TSUGAWA ${ }^{3}$ and NARIKAZU BOKU ${ }^{1}$ \\ Departments of ${ }^{1}$ Clinical Oncology and ${ }^{2}$ Nursing; ${ }^{3}$ Division of Breast and Endocrine Surgery, Department of Surgery, \\ St. Marianna University School of Medicine, Kawasaki 216-8511, Japan
}

Received August 18, 2015; Accepted January 18, 2016

DOI: $10.3892 / \mathrm{mco} .2016 .769$

\begin{abstract}
The incidences of infusion site adverse events in chemotherapy regimens, including anthracyclines with either fosaprepitant or aprepitant as the anti-emetic, were not highlighted in the randomized trial comparing aprepitant and fosaprepitant. The present retrospective analysis was performed in breast cancer patients receiving anthracycline-containing chemotherapy, a combination of epirubicin and cyclophosphamide with or without 5-fluorouracil as the adjuvant or neoadjuvant, at the outpatient infusion center of St. Marianna University Hospital (Kawasaki, Japan). Infusion site adverse events were retrospectively compared between the 3 months prior to and three months following switching from 3 day oral administration of aprepitant to intravenous infusion of fosaprepitant. A total of 62 patients were included in the aprepitant group and 38 in the fosaprepitant group. Of these patients, $26(42 \%)$ in the aprepitant group and 36 patients (96\%) in the fosaprepitant group experienced any grade of infusion site adverse events at least once $(\mathrm{P}<0.001)$. As an anti-emetic treatment for chemotherapy using anthracyclines, fosaprepitant may be associated with a higher risk of infusion site adverse events compared with aprepitant.
\end{abstract}

Correspondence to: Dr Narikazu Boku, Department of Clinical Oncology, St. Marianna University School of Medicine, 2-16-1 Sugao, Miyamae-ku, Kawasaki 216-8511, Japan E-mail: n.boku@marianna-u.ac.jp

Key words: infusion site adverse events, fosaprepitant, anthracyclines

\section{Introduction}

Aprepitant, a neurokinin-1 receptor antagonist, is effective for the prevention of both acute and delayed chemotherapy-induced nausea and vomiting (CINV) in patients receiving highly emetic chemotherapy (HEC) and moderately emetic chemotherapy (MEC), when administered with a serotonin receptor antagonist and dexamethasone (1-4). In numerous anti-emetic treatment guidelines, aprepitant is recommended for patients receiving HEC and MEC (5). Fosaprepitant dimeglumine, a phosphorylated analog of aprepitant, is rapidly converted to aprepitant following intravenous administration. A randomized control trial demonstrated that a one-time intravenous infusion of fosaprepitant was non-inferior to 3 day oral administration of aprepitant, when combined with dexamethasone and ondansetron (6). Based on these results, fosaprepitant has been widely replacing aprepitant in the prophylactic treatment of CINV as a result of its convenience.

However, it has been recently reported that the incidences of infusion site adverse events, including infusion site pain, swelling, erythema, induration and itching, have increased, particularly in chemotherapy regimens including anthracyclines, by using fosaprepitant compared with aprepitant. These problems were not highlighted in the randomized trial comparing aprepitant and fosaprepitant (6). Currently, no consensus regarding the risk of infusion site adverse events associated with fosaprepitant exist and major guidelines do not mention this problem.

At St. Marianna University (Kawasaki, Japan), all chemotherapy regimens, including anti-emetic prophylactic treatments, are approved based on the evidence level following review by the cancer treatment committee, and are registered in the computer ordering system. Oral aprepitant in HEC regimens was uniformly replaced by fosaprepitant soon after fosaprepitant was approved in Japan. In the present retrospective analysis, infusion site adverse events were retrospectively compared between the periods of 3 months prior to and 3 months following switching from aprepitant to 
Table I. Characteristics of patients.

\begin{tabular}{lccc}
\hline Characteristic & Aprepitant group & Fosaprepitant group & P-value \\
\hline Age & & & \\
$\quad$ Median (range) & $52(30-75)$ & $47(31-66)$ & \\
Regimen & $28(45 \%)$ & $11(29 \%)$ & 0.107 \\
EC & $34(55 \%)$ & $27(71 \%)$ & \\
FEC & $53(85 \%)$ & $32(84 \%)$ & 0.863 \\
Line & $9(15 \%)$ & $6(16 \%)$ & \\
NAC & & & \\
ADJ &
\end{tabular}

EC, epirubicin/cyclophosphamide; FEC, epirubicin/cyclophosphamide/fluorouracil; NAC, neoadjuvant chemotherapy; ADJ, adjuvant chemotherapy.

fosaprepitant in chemo-naïve breast cancer patients receiving anthracycline-containing chemotherapy.

\section{Materials and methods}

The subjects of the present study were chemo-naïve breast cancer patients who were administered anthracycline-containing chemotherapy, a combination of epirubicin and cyclophosphamide (EC), or EC with 5-fluorouracil (FEC) as the adjuvant or neoadjuvant, at the outpatient infusion center of St. Marianna University Hospital. This treatment was provided between the 3 months prior to (Dece mber $13^{\text {th }}$ 2011-March 12 $2^{\text {th }} 2012$ ) and the 3 months following (March 13 ${ }^{\text {th }} 2012$ and June 12 ${ }^{\text {th }} 2012$ ) switching from 3 day oral administration of aprepitant to intravenous infusion of fosaprepitant (dissolved in $100 \mathrm{ml}$ physiological saline and injected over $30 \mathrm{~min}$ ). FEC and EC were planned to be administered four times in total. The treatment schedule of EC comprised epirubicin $\left[90 \mathrm{mg} / \mathrm{m}^{2} ; 10 \mathrm{~min}\right.$ intravenous (IV) infusion] plus cyclophosphamide $\left(600 \mathrm{mg} / \mathrm{m}^{2} ; 30 \mathrm{~min}\right.$ IV infusion) on day 1 every 3 weeks, and that of FEC was epirubicin (100 mg/m² $10 \mathrm{~min}$ IV infusion) and cyclophosphamide $\left(500 \mathrm{mg} / \mathrm{m}^{2} ; 30 \mathrm{~min}\right.$ IV infusion), followed by fluorouracil (500 mg/m²; $15 \mathrm{~min}$ IV infusion) on day 1 every 3 weeks. The dose of chemotherapy may be reduced and/or delayed at the physician's discretion considering the adverse events. Needle insertion was performed by specially trained nurses approved by the director of the hospital, or by skillful doctors. The anti-emetic treatment was based on granisetron hydrochloride (1 mg), ranitidine (50 mg), and dexamethasone $(6.6 \mathrm{mg})$ on day 1 by IV infusion and oral dexamethasone $(8 \mathrm{mg}$ ) on days 2 and 3 . The subjects were divided into aprepitant $(n=62)$ and fosaprepitant $(n=38)$ groups. The patients whose anti-emetic treatment was switched from aprepitant to fosaprepitant and those who received chemotherapy via the central venous route were excluded from the present study. In the fosaprepitant group, the anti-emetic could be changed to aprepitant in the case of infusion site adverse events.

Evaluation of infusion site adverse events. As routine clinical practice at the outpatient infusion center of St. Marianna University Hospital, each patient reported the grade (0-4) of symptomatic adverse events by checking daily on the dedicated forms specific to each chemotherapy regimen, focusing on fatigue, anorexia, nausea, vomiting, dysgeusia, mucositis, fever, constipation, diarrhea, alopecia, palmar-plantar erythrodysesthesia syndrome, nail loss, injection site reaction, gait disturbance, pain and anxiety. Patients receiving chemotherapy at the outpatient infusion center were monitored by the special nurses, paying particular attention to the infusion site adverse events, and all patients were evaluated according to the CTCAE ver. 4.0 (http://ctep. cancer.gov/protocolDevelopment/electronic_applications/docs/ ctcae_4_with_lay_terms.pdf) and recorded on the electronic medical chart routinely.

\section{Results}

In total, 102 breast cancer patients commenced the neoadjuvant or adjuvant chemotherapy during the above period, of whom two were excluded from the present study as a result of switching from fosaprepitant to aprepitant during the chemotherapy, in spite of the absence of infusion site adverse events. Finally, 62 were included in the aprepitant group and the remaining 38 were in the fosaprepitant group. Table I describes the background of the patients in each group. While no differences were observed in age and treatment lines, adjuvant or neoadjuvant, the proportion of the patients receiving EC regimens was slightly higher in the aprepitant group (45\%) compared with the fosaprepitant group (29\%; $\mathrm{P}=0.107)$.

All patients in each group completed the four planned cycles of chemotherapy. It was necessary to reduce of amount of chemotherapy administered to five patients $(8 \%)$ in the aprepitant group: Two with febrile neutropenia (FN) and three with grade 2 nausea and vomiting. In the fosaprepitant group, it was necessary to reduce the amount of chemotherapy administered to seven patients (18\%); three with FN, two with grade 2 fatigue, one with grade 3 neutropenia and one with grade 2 liver dysfunction.

A total of 26 patients (42\%) in the aprepitant group and 36 patients $(96 \%)$ in the fosaprepitant group experienced infusion site adverse events of any grade at least once $(\mathrm{P}<0.001)$. Observed infusion site adverse events were pain, 
Table II. Infusion site adverse events profiles.

Adverse event

Aprepitant group

Fosaprepitant group

Infusion site pain

Swelling

Erythema

Venous hardening/induration

Itching sensation

Local scarring

Flexion difficult

Heat sensation

Phlebitis

$\begin{array}{rr}26 & 35 \\ 0 & 12 \\ 0 & 15 \\ 0 & 11 \\ 0 & 2 \\ 1 & 2 \\ 0 & 5 \\ 2 & 5 \\ 0 & 6\end{array}$

Table III. Number of cycles when the first occurrence of any grade infusion site adverse events were observed.

swelling, erythema, induration, itching, scar, difficulty in flection, heat sensation and phlebitis (Table II). Grade 2 infusion site adverse events were observed more frequently in the fosaprepitant group (61\%) compared with in the aprepitant group $(7 \%$; $\mathrm{P}<0.001)$. A total of $3 / 26$ patients in the aprepitant group and $26 / 36$ patients in the fosaprepitant group who experienced any infusion site adverse events required treatment for them. Table III shows the number of cycles when the first occurrence of infusion site adverse events of any grade were observed.

In the fosaprepitant group, $45 \%$ of the patients experienced their first infusion site adverse event in the first cycle and $>3 / 4(78 \%)$ of those did so within the first two cycles. A total of 17/36 patients in the fosaprepitant group continued fosaprepitant even after experiencing infusion site adverse events, and similar adverse events were observed in 12 patients. Owing to the infusion site adverse events, fosaprepitant was discontinued in 19 patients in the fosaprepitant group, and aprepitant or palonosetron were subsequently used in 7 and 12 patients, respectively, with similar adverse events being observed in 5/19 patients. In 14 out of these 19 patients (74\%), the infusion site adverse events improved or disappeared following the discontinuation of fosaprepitant. Furthermore, a central venous catheter was inserted into three patients and no further infusion site adverse events were observed in these patients.

No nausea was reported by $10 \%$ of the patients in the aprepitant group compared with $18 \%$ of the patients in the fosaprepitant group. No vomiting was reported by $85 \%$ of the patients in the aprepitant group compared with $76 \%$ of the patients in the fosaprepitant group. No significant differences were observed between the two groups.

\section{Discussion}

Although the incidence of infusion site adverse events has been reported to be higher in fosaprepitant treatment compared with in aprepitant, particularly with chemotherapy using anthracyclines, the precise mechanism and the interaction between fosaprepitant and anthracyclines remain to be elucidated. In previous reports focusing on anthracycline-containing regimens, the incidences of infusion site adverse events range between 35 and 67\% (7-9), while adverse events of any grade and grade 2 were as high as 95 and $61 \%$, respectively, in the present study. The incidences of infusion site adverse

\begin{tabular}{lcc}
\hline Cycle & $\begin{array}{c}\text { Aprepitant group } \\
(\mathrm{n}=26)\end{array}$ & $\begin{array}{c}\text { Fosaprepitant group } \\
(\mathrm{n}=36)\end{array}$ \\
\hline 1 & $6(23 \%)$ & $16(45 \%)$ \\
2 & $5(19 \%)$ & $12(33 \%)$ \\
3 & $10(39 \%)$ & $5(14 \%)$ \\
4 & $5(19 \%)$ & $3(8 \%)$ \\
\hline
\end{tabular}

events $(42 \%)$ in the present study were higher compared with those reported previously by $\sim 10 \%$, even in the aprepitant group. In the present study, aprepitant was switched to fosaprepitant uniformly by changing the chemotherapy regimens registered in the computer ordering system, and the infusion site reactions were compared prior to and following switching. This means that there were very small patient selection biases in either arm, while other retrospective reports may exhibit certain selection biases. It was previously reported that prior chemotherapy may increase the risk of infusion site adverse events (10). However, since all the subjects in the present study were chemo-naïve, this may lead to a precise evaluation of infusion site adverse events without being affected by previous chemotherapy. However, special nurses dedicated to the outpatient infusion center checked the patient report forms of adverse events, and paid special attention to checking and recording infusion site adverse events. This evaluation system may overestimate the infusion site adverse events compared with previous reports, many of which were based on the physician's medical records comparing simultaneously between aprepitant and fosaprepitant. When continuing fosaprepitant after the first occurrence of infusion site adverse events, they reappeared in $74 \%$ of the patients, while most of the patients who discontinued fosaprepitant completed the planned chemotherapy without further infusion site adverse events. No differences were observed in other adverse events between the aprepitant group and the fosaprepitant group. Therefore, the present retrospective analysis provided evidence that fosaprepitant should be replaced by other anti-emetic agents, including aprepitant. Inserting a central venous catheter may be another option to prevent infusion site adverse events when using fosaprepitant. 
In conclusion, as an anti-emetic treatment for chemotherapy using anthracyclines, fosaprepitant may be associated with a higher risk of infusion site adverse events compared with aprepitant.

\section{References}

1. Hesketh PJ, Warr DG, Street JC and Carides AD: Differential time course of action of 5-HT3 and NK1 receptor antagonists when used with highly and moderately emetogenic chemotherapy (HEC and MEC). Support Care Cancer 19: 1297-1302, 2011.

2. Herrstedt J, Muss HB, Warr DG, Hesketh PJ, Eisenberg PD, Raftopoulos H, Grunberg SM, Gabriel M, Rodgers A, Hustad CM, et al: Efficacy and tolerability of aprepitant for the prevention of chemotherapy-induced nausea and emesis over multiple cycles of moderately emetogenic chemotherapy. Cancer 104: 1548-1555, 2005.

3. Herrington JD, Jaskiewicz AD and Song J: Randomized, placebo-controlled, pilot study evaluating aprepitant single dose plus palonosetron and dexamethasone for the prevention of acute and delayed chemotherapy-induced nausea and vomiting. Cancer 112: 2080-2087, 2008.

4. Rapoport BL, Jordan K, Boice JA, Taylor A, Brown C, Hardwick JS, Carides A, Webb T and Schmoll HJ: Aprepitant for the prevention of chemotherapy-induced nausea and vomiting associated with a broad range of moderately emetogenic chemotherapies and tumor types: A randomized, double-blind study. Support Care Cancer 18: 423-431, 2010.
5. Basch E, Prestrud AA, Hesketh PJ, Kris MG, Feyer PC, Somerfield MR, Chesney M, Clark-Snow RA, Flaherty AM, Freundlich B, et al: Antiemetics: American society of clinical oncology clinical practice guideline update. J Clin Oncol 29: 4189-4198, 2011.

6. Grunberg S, Chua D, Maru A, Dinis J, DeVandry S, Boice JA, Hardwick JS, Beckford E, Taylor A, Carides A, et al: Single-dose fosaprepitant for the prevention of chemotherapy-induced nausea and vomiting associated with cisplatin therapy: Randomized, double-blind study protocol-EASE. J Clin Oncol 29: 1495-1501, 2011.

7. Leal AD, Kadakia KC, Looker S, Hilger C, Sorgatz K, Anderson K, Jacobson A, Grendahl D, Seisler D, Hobday T and Loprinzi CL: Fosaprepitant-induced phlebitis: A focus on patients receiving doxorubicin/cyclophosphamide therapy. Support Care Cancer 22: 1313-1317, 2014.

8. Sato Y, Kondo M, Inagaki A, Komatsu H, Okada C, Naruse K, Sahashi T, Kuroda J, Ogura H, Uegaki S, et al: Highly frequent and enhanced injection site reaction induced by peripheral venous injection of fosaprepitant in anthracycline-treated patients. J Cancer 5: 390-397, 2014.

9. Fujii T, Nishimura N, Urayama KY, Kanai H, Ishimaru H, Kawano J, Takahashi O, Yamauchi $\mathrm{H}$ and Yamauchi T: Differential impact of fosaprepitant on infusion site adverse events between cisplatin and anthracycline-based chemotherapy regimens. Anticancer Res 35: 379-383, 2015.

10. Lundberg JD, Crawford BS, Phillips G, Berger MJ and Wesolowski R: Incidence of infusion-site reactions associated with peripheral intravenous administration of fosaprepitant. Support Care Cancer 22: 1461-1466, 2014. 